\title{
Abstraction of significant temporal features of voltage Sags recorded in a $25 \mathrm{kV}$ substation
}

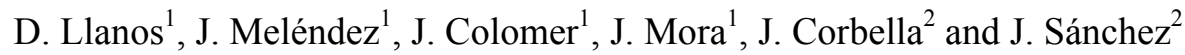 \\ ${ }^{1}$ Control Engineering and Intelligent Systems Group - eXiT \\ University of Girona \\ Av. Lluis Santalo s/n. Building P.IV - Girona (Spain) \\ phone:+34 972 418391, fax:+34 972418 97601, e-mail: dllanosr@eia.udg.es \\ quimmel@eia.udg.es colomer@eia.udg.es jjmora@eia.udg.es \\ ${ }^{2}$ ENDESA \\ FJCorbella@enher.es jslosada@fecsa.es
}

\begin{abstract}
.
The existing methods to characterise voltage sags use the lowest of the three voltages and the longest duration. Basic quantitative | qualitative voltage sag attributes have been obtained from recorded waveforms in order to increase the number of measures to characterise a voltage sag. Also a method to compare voltage sags have been proposed and applied in sags recorded in a $25 \mathrm{kV}$ substation.
\end{abstract}

\section{Key words}

Characterisation, sags, symmetrical components, DTW, Case Based Reasoning.

\section{Introduction}

The existence of incidences registers (utility data bases) with information related with the causes, location and protective relay trips that can be associated with waveform registers have motivated this work. The goal is to characterise voltage sags in order to establish a similarity criteria among them. This is the first step to build a diagnostic system based on the reuse of past experiences (diagnosed sags) according to the Case Based Reasoning (CBR) methodology. Application of CBR to diagnosis is commonly known as Case Based Diagnosis.

Case Based Reasoning (CBR) is a problem solving methodology based on the reuse of previous experiences. It is based on Schank's dynamic memory models [8] and the basic idea is focused on the hypothesis that "similar problems have similar solutions" [9]. CBR methodology proposes a four-step cycle (Retrieve, Reuse, Revise and Retain) also known as the 4R-cycle (Figure 1). It basically consists in Retaining experiences as cases for a further Reuse (submitted to a Revision procedure). Cases are registers containing a description of a problem, "sag symptoms", and its solution, "sag diagnostic". The aim is to reuse these cases for solving new problems by analogy. In presence of a new problem, the basic procedure consists of Retrieving analogue cases (sags), according to their description (attributes defining symptoms), and reusing their solutions (diagnostic). A wider introduction and foundation of CBR can be consulted in [10] and [11].

In this work main emphasis is put in defining similarity criteria to implement the Retrieval task based on significant features extracted from sags waveforms. Reuse is reduced to a simple copy of the retrieved diagnostic.

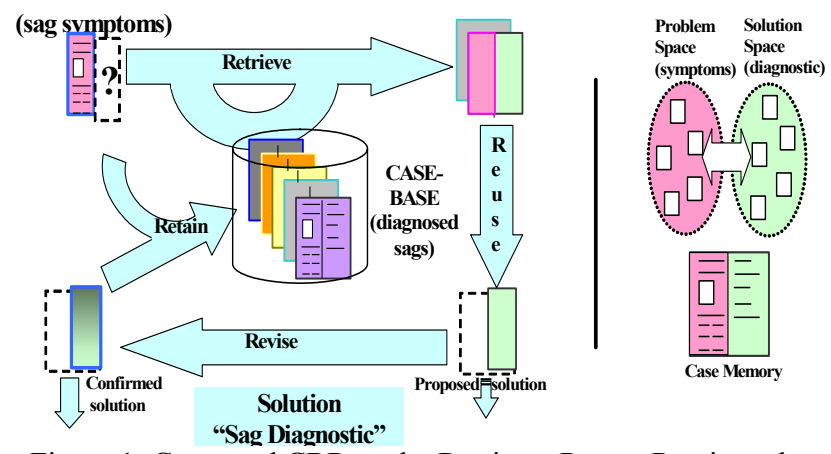

Figure 1: Cases and CBR cycle: Retrieve, Reuse, Retain and

Revise.

Significant attributes to characterise sags are proposed in the text involving both temporal and phasorial description of them. The aim is to improve simple definition of sags that only give relations among global drop magnitude and duration obtained from the rms values.

Standard definition of sags ( $[1][2][3][4][5])$ is based on the minimum rms value obtained during the event and its duration is the time interval between the instant when rms voltage crosses the voltage sag threshold (usually $90 \%$ of normal voltage) and the instant when it returns to normal level (a three-phase unbalanced voltage sag is shown in Figure 2). There are obvious limitations to use this definition to compare (distinguish or/and identify 
similar ones) sags, e.g. it neglects the phase-angle jump [2][6] and the post-fault voltage evolution [7].

Two complementary methods are compared in the paper with this aim. The first one is based on using temporal attributes obtained from the instantaneous rms value (computed in a one-cycle time-window) of each phase. The second method uses a Dynamic Time Warping (DTW) algorithm in order to measure the distance between the characteristic voltage of sags. Both methods are applied in a pruned case based that only contains typological equal sags (See section 2.C).
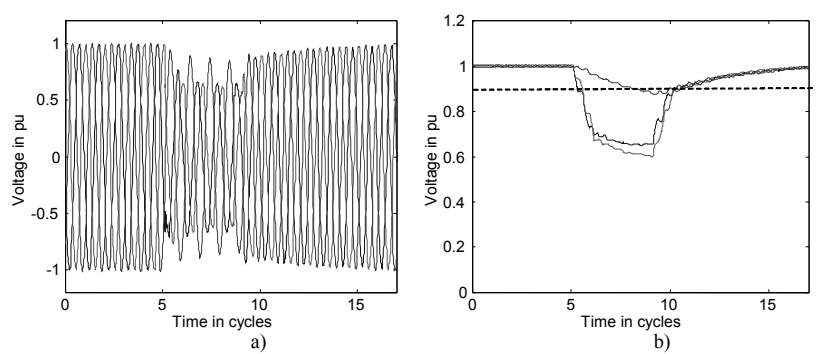

Figure 2 a) Example of a three-phase unbalanced voltage sag. b) rms voltage.

This paper is organised as follows. Sag attributes are presented in section 2. In section 3, two methods to measure similarity between sags in order to retrieve the most similar ones from a case-base are proposed. In section 4, a numerical example is presented by using voltage sags recorded in a $25 \mathrm{kV}$ substation during a period of 10 months. Finally, conclusions and futures work are summarised in section 5 .

\section{Significant voltage sag attributes}

Voltage sags have been characterized in this work by using temporal and phasorial attributes. Temporal attributes are obtained from measurements of the duration and magnitude whereas phasorial attributes are represented qualitatively according to the method presented in [2].

The main idea is to have enough attributes in order to compare voltage sags for an efficient retrieve based on similarities.

Sag attributes have been divided in three-phase and single-phase attributes and voltage sag characterization based on symmetrical components [13].

\section{A. Three-phase temporal attributes}

Three-phase temporal attributes depicted in figure 3, are:

1) Three-phase sag magnitude: Defined as the maximum drop of voltage of three-phase power system during the sag.

2) Three- phase sag duration: Defined, as the maximum time during the rms voltage in a threephase power system, is lower to 0.9 p.u.

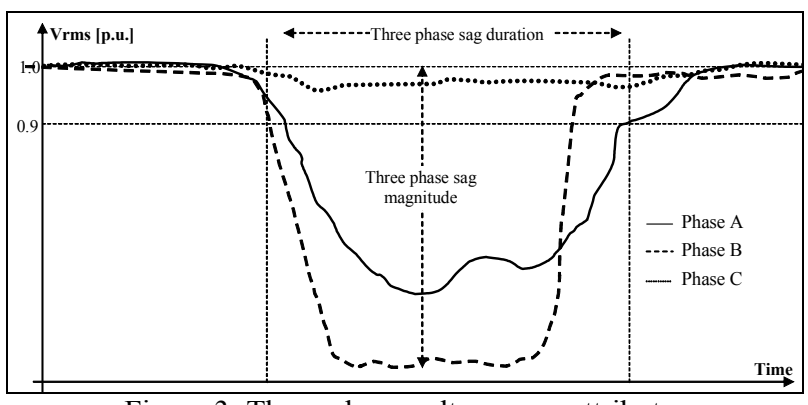

Figure 3: Three-phase voltage sags attributes

\section{B. Single-phase temporal attributes}

Single-phase temporal attributes depicted in figure 4, are:

1) Single-phase sag magnitude (h): Maximum voltage drops in every single phase of the power system.

2) Single-phase sag duration: Maximum time during the rms voltage is lower to 0.9 p.u. in every single phase of the power system .

3) Voltage fall slope: Slope at the beginning of the sag until a steady state is reached within a $2 \%$ band [12].

4) Voltage recovery slope: Slope in the end of the sag until a steady state is reached within a $2 \%$ band [12].

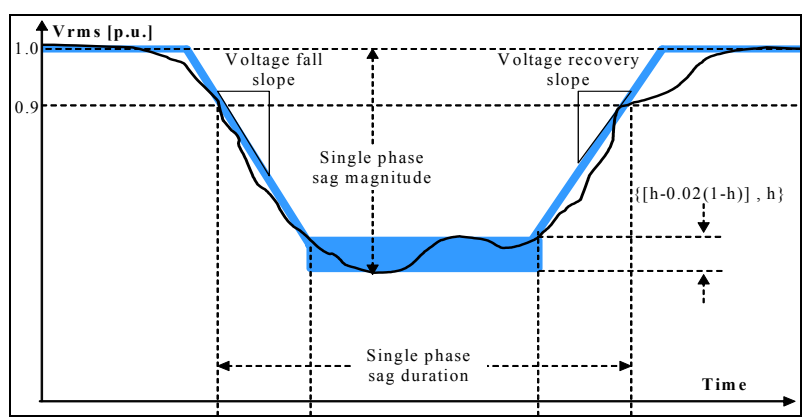

Figure 4: Single-phase voltage sags attributes

\section{Voltage sag characterisation based on symmetrical components}

A technique proposed in [2] to characterise sags, has been applied, which enables a characterization through one complex voltage, without significant loss of information. The method is based on the decomposition of the voltage phasors in symmetrical components [13]. Positive-sequence voltage $\vec{V}_{1}$, negative-sequence voltage $\vec{V}_{2}$ and zero-sequence voltage $\vec{V}_{0}$ are calculated from the complex phase voltages $\vec{V}_{a}, \vec{V}_{b}$ and $\vec{V}_{c}$ as follows:

$$
\left[\begin{array}{c}
\vec{V}_{0} \\
\vec{V}_{1} \\
\vec{V}_{2}
\end{array}\right]=\frac{1}{3}\left[\begin{array}{ccc}
1 & 1 & 1 \\
1 & a & a^{2} \\
1 & a^{2} & a
\end{array}\right]\left[\begin{array}{l}
\vec{V}_{a} \\
\vec{V}_{b} \\
\vec{V}_{c}
\end{array}\right]
$$

where

$$
a=-\frac{1}{2}+\frac{1}{2} j \sqrt{3}
$$

The voltage sag type indicates which phases are involved in the event. The seven basic types are given in figure 5 . Balanced voltage sag (type A) is due to an equal drop in the values of voltage in the three-phases. Unbalanced voltage sags (types C and D) depend on the phases 
involved. The C-types are voltage drops between two phases: type $\mathrm{Ca}$ is a voltage drop between phases $\mathrm{b}$ and $\mathrm{c}$, type $\mathrm{Cb}$ between phases a and $\mathrm{c}$, and type $\mathrm{Cc}$ between phases $a$ and $b$. The D-types are voltage drops in one phase: type $\mathrm{Da}$ is a voltage drop in phase a, type $\mathrm{Db}$ in phase b, and type Dc in phase c. [12].
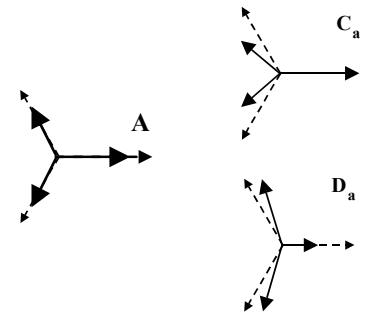

a)

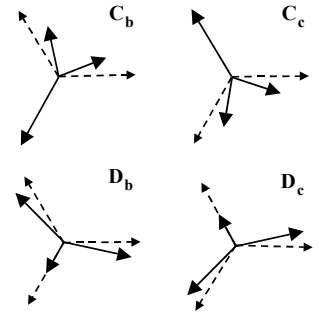

b)
Figure 5, a) Three-phase balance voltage sag, b) six types of three-phase unbalanced voltage sags.

The voltage sag type is found from the angle between positive-sequence voltage $\vec{V}_{1}$ and negative-sequence voltage $\vec{V}_{2}$. The classification method is described in more detail in [2] and summarised in the next subsection.

$$
k=\operatorname{round}\left(\frac{\operatorname{angle}\left(\vec{V}_{2}, 1-\vec{V}_{1}\right)}{60^{\circ}}\right)
$$

where,

$$
\begin{array}{lll}
\mathrm{k}=0 \text { : type Ca } & \mathrm{k}=1 \text { : type } \mathrm{Dc} & \mathrm{k}=2 \text { : type } \mathrm{Cb} \\
\mathrm{k}=3 \text { : type } \mathrm{Da} & \mathrm{k}=4 \text { : type } \mathrm{Cc} & \mathrm{k}=5 \text { : type } \mathrm{Db}
\end{array}
$$

Knowing the voltage sag type, the negative-sequence voltage can be calculated back to the corresponding value for prototype voltage sag:

$$
\vec{V}_{2}^{\prime}=\vec{V}_{2} e^{-j k 60^{\circ}}
$$

where $k$ is obtained according to (2) and the negative sequence voltage of the measured voltage sag. Characteristic voltage $\vec{V}$ and PN-factor $\vec{F}$ are obtained from:

$$
\begin{aligned}
& \vec{V}=\vec{V}_{1}-\vec{V}_{2}^{\prime} \\
& \vec{F}=\vec{V}_{1}+\vec{V}_{2}^{\prime}
\end{aligned}
$$

The voltage magnitude is defined as the absolute value of the characteristic voltage. The voltage phase angle as the argument of the characteristic voltage.

The proposal is to use characteristic complex voltage and PN-factor to characterise three-phase unbalanced voltage sags. The proposed method for classification and characterisation consists of a number of steps. It is assumed that time-domain sampled data is available for the three phases including at least two cycles preevent voltages [2].
- Determine voltage phasors for the three phase voltages by using a DFT/FFT (Discrete Fourier Transform) algorithm. The voltage frequency is used to obtain the phase shift between the during-event and the pre-event voltages.

- Obtain positive, negative and zero-sequence voltages (1).

- Determine if the voltage sag is balanced or unbalanced from the magnitude of the negative sequence voltage compared to the positive sequence voltage.

- For balanced voltage sag type is A and the characteristic voltage equals the positive sequence voltage.

- For unbalanced voltage sag type is determined from positive and negative-sequence voltages by using expression (2), characteristic voltage and PN-factor are obtained by using expression (4).

- A balanced voltage sag is fully characterized through the characteristic voltage.

- An unbalanced voltage sag is fully characterized through voltage sag type, characteristic voltage, $\mathrm{PN}$-factor and zero-sequence voltage.

- The characteristic magnitude is obtained as the absolute value of the characteristic voltage.

$D$ Attributes summary used in voltage sag characterisation

1) Temporal attributes: as voltage sag duration, it helps to locate and establish the classes and voltages sags relationships, in transport and distribution systems. This was concluded using the knowledge provided by the electric facility's engineers, then the fault location scheme in figure 6 was proposed.

The times showed in figure 6 include the protective system delay and the breaker interrupting time (3-5 cycles), therefore this time is used as reference to the voltage sag duration.

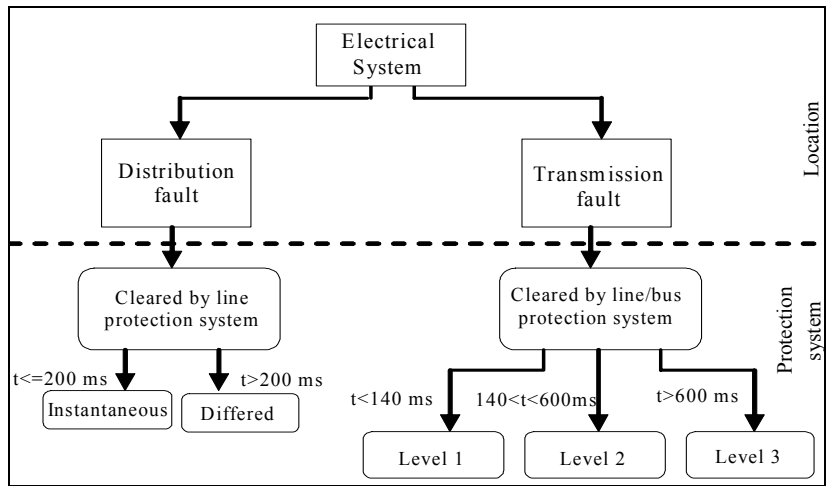

Figure 6: Fault location scheme related to protection system

2) Voltage sag type gives information about the origin of the fault, type A due to three-phase 
faults, type $\mathrm{C}$ and $\mathrm{D}$ due to single-phase and phase-to-phase faults.

3) The characteristic voltage is comparable to the rms voltage for single-phase measurements and should be used to compare global shape of sags.

4) According to [2], it is found that PN-factor is very close to unit in transmission systems and less than unit in distribution systems, due to the effect of induction motor load. However, even in distribution systems, the PN-factor is rarely less than $90 \%$, in absolute value.

\section{Proposed method to measure voltage sags similarities}

The main idea in this section is to compare sags in order to retrieve the most similar voltage sags from a case-base. A metric to define similarities between sags $S_{A}$ and $S_{B}$, $\operatorname{DIST}\left(\mathrm{S}_{\mathrm{A}}, \mathrm{S}_{\mathrm{B}}\right)$; is proposed in two steps. First determine the voltage sag type using symmetrical components. For the second step two methods have been proposed: one measuring distance between temporal attributes by using Manhattan distance and the second measuring distance between characteristic voltages by using Dynamic Time Warping. Proposed method is described as follows:

\section{A. Determine the voltage sag type}

Determine the voltage sag type using the classification algorithm based on symmetrical components proposed in section 2 .

The objective is to reduce the search area in the voltage sag case-base. It is not necessary to compare a new sag with a voltage sag classified as different type.

Useful information for the diagnosis, as origin of the fault (type A due to three-phase faults, type C and D due to single-phase and phase-to-phase faults).

\section{B. Temporal attributes distance method.}

Once selected the voltage sags with the same type, comparison continues with temporal attributes. A metric have been proposed to compare similarities between two sags $S_{A}$ and $S_{B}$ as is depicted in figure 7 .

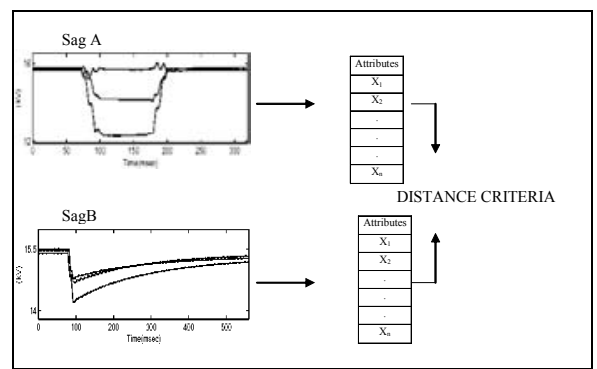

Figure 7. Similarities between voltage sags.
A sag $\mathrm{S}$, is defined by the vector of $\mathrm{n}$ attributes $\left(\mathrm{X}_{\mathrm{i}}\right.$, with $i=1 . . n)$. Thus, the distance between two sags $\left(S_{A}\right.$ and $\left.S_{B}\right)$ can be computed as a weighted addition of local distances (equation (5)) between normalised attributes.

$$
\operatorname{DIST}\left(S_{A}, S_{B}\right)=\sum_{i=1}^{n} W_{i} \operatorname{dist}\left(\overline{X_{i}^{A}}, \overline{X_{i}^{B}}\right)
$$

With:

$$
\operatorname{dist}\left(\overline{X_{i}^{A}}, \overline{X_{i}^{B}}\right)=\left|\overline{X_{i}^{A}}-\overline{X_{i}^{B}}\right|
$$

Normalisation is performed according to the range of each attribute $\left(\mathrm{X}_{\mathrm{i} \text { min }}-\mathrm{X}_{\mathrm{i} \text { max }}\right)$. The following expression has been used with this purpose:

$$
0<\frac{X_{i}-X_{i \min }}{X_{i \max }-X_{i \min }}<1
$$

Voltage sag magnitude and voltage sag duration have been normalised according to the categories and typical characteristics of power system electromagnetic phenomena [14], where voltages sag (included in short duration variations category) is defined with a typical duration of (0.5-30 cycles) and typical voltage magnitude of (0.1-0.9 p.u.).

\begin{tabular}{|c|c|c|c|c|c|}
\hline $\begin{array}{c}\text { Three-phase temporal } \\
\text { Attributes }\end{array}$ & \multicolumn{4}{|c|}{ Single-phase temporal Attributes } \\
\hline Magnitude & Duration & Magnitude & Duration & Fall slope & Recovery slope \\
\hline & $\begin{array}{c}0.5-30 \\
\text { cycles }\end{array}$ & $*$ & $*$ & $*$ & $*$ \\
\hline
\end{tabular}

Table 1.Reference values to normalise temporal attributes *: those values have been selected from the maxim and minimum of real measures.

\section{Dynamic Time Warping.}

Most of algorithms that operate with time series of data use the Euclidean distance or some variation. However, Euclidean distance could produce an incorrect measure of similarity because it is very sensitive to small distortions in the time axis.

A method that tries to solve this inconvenience is Dynamic Time Warping (DTW), this technique uses dynamic programming (Sakoe and Chiba, 1978; Silverman, 1990) to align time series with a given template so that the total distance measure is minimised (Figure. 8). DTW has been widely used in word recognition to compensate the temporal distortions related to different speeds of speech. Next, a brief notion of DTW is described.

Given two sags $\mathrm{S}_{\mathrm{A}}, \mathrm{S}_{\mathrm{B}}$ described by its characteristic voltage $\vec{V}_{A}(t), \vec{V}_{B}(t)$, of length $m$ and $n$ respectively, the aim objective is to measure distance between them, $\operatorname{DIST}\left(\mathrm{S}_{\mathrm{A}}, \mathrm{S}_{\mathrm{B}}\right)=\operatorname{DTW}\left(\vec{V}_{A}(t), \vec{V}_{B}(t)\right)$.

$$
\begin{aligned}
& \vec{V}_{A}(t)=\mathrm{v}_{\mathrm{A} 1}, \mathrm{v}_{\mathrm{A} 2}, \ldots, \mathrm{v}_{\mathrm{Ai}}, \ldots, \mathrm{v}_{\mathrm{Am}} \\
& \vec{V}_{B}^{A}(t)=\mathrm{v}_{\mathrm{B} 1}, \mathrm{v}_{\mathrm{B} 2}, \ldots, \mathrm{v}_{\mathrm{Bj}}, \ldots, \mathrm{v}_{\mathrm{Bn}}
\end{aligned}
$$

To align the two sequences, DTW will find a sequence $S$ of $\mathrm{k}$ points on a m-by-n matrix that minimise the distance between the two sequences. 


$$
\mathrm{S}=\mathrm{s}_{1}, \mathrm{~S}_{2}, \ldots, \mathrm{S}_{\mathrm{k}} \quad \max (\mathrm{m}, \mathrm{n}) \leq \mathrm{k} \leq \mathrm{m}+\mathrm{n}
$$

The path is extracted by evaluating the cumulative distance $\mathrm{D}(\mathrm{i}, \mathrm{j})$ as the sum of the local distance $\mathrm{d}\left(\mathrm{x}_{\mathrm{i}}, \mathrm{y}_{\mathrm{j}}\right)$ in the current cell and the minimum of the cumulative distances in the previous cells. This can be expressed as:

$$
D(i, j)=d\left(v_{A i}, v_{B j}\right)+\min [D(i-1, j-1), D(i-1, j), D(i, j-1)](10)
$$

This method is described in more detail in literature [15].
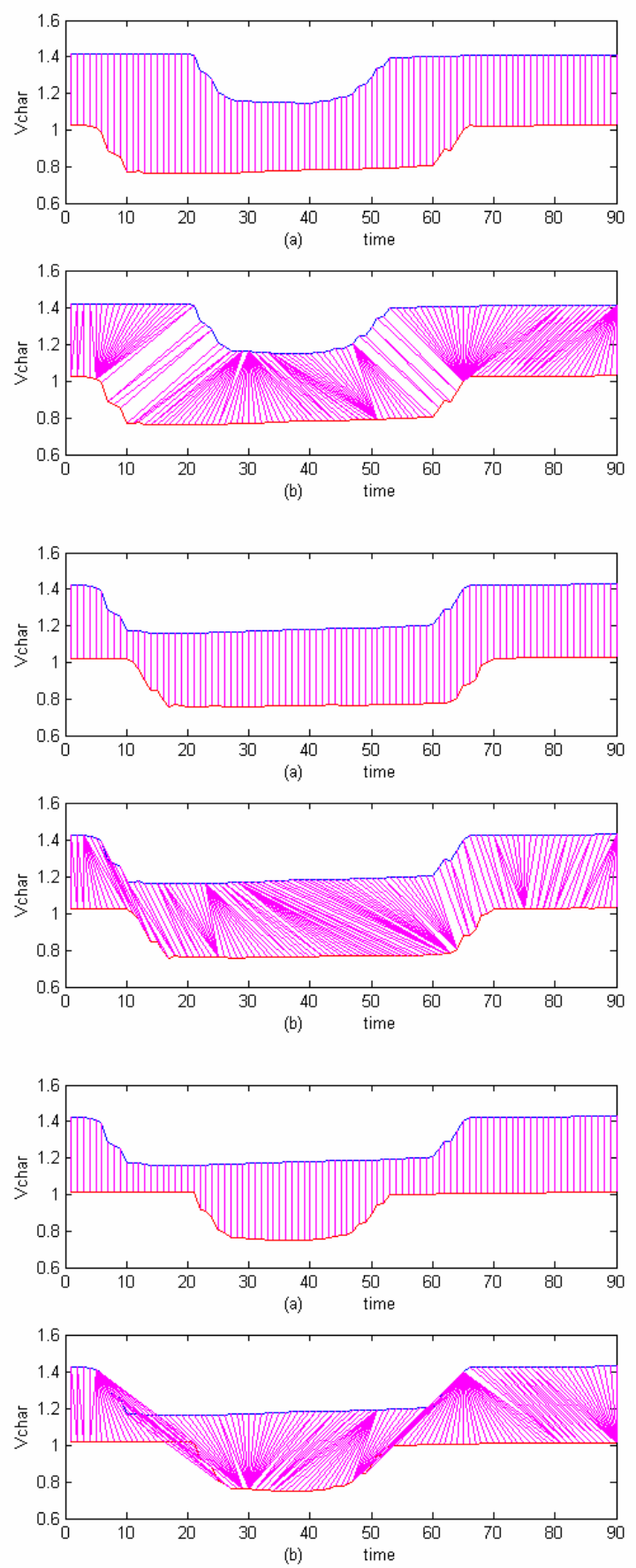

Figure. 8 Two similar characteristic voltage sag . a) The Euclidean distance b) DTW

In figure 8a) The Euclidean distance produce a pessimistic result of similarity since the signals are not aligned in time. In figure 8b) DTW find an alignment that allows a correct measure of similarity, lines between shapes show the point compared in each waveform.

\section{Application in a $25 \mathrm{kV}$-Electrical substation}

Spanish Electrical Facility (Endesa Distribution SL) has provided voltage sags in a $25 \mathrm{kV}$ distribution substation. 50 voltage sags were recorded and chosen to apply this method. They were separated in two subgroups according to the location of the fault (transmission/ distribution). Additional description related with the origin is presented.

Temporal attributes of sags recorder in the substation are depicted in table 2.

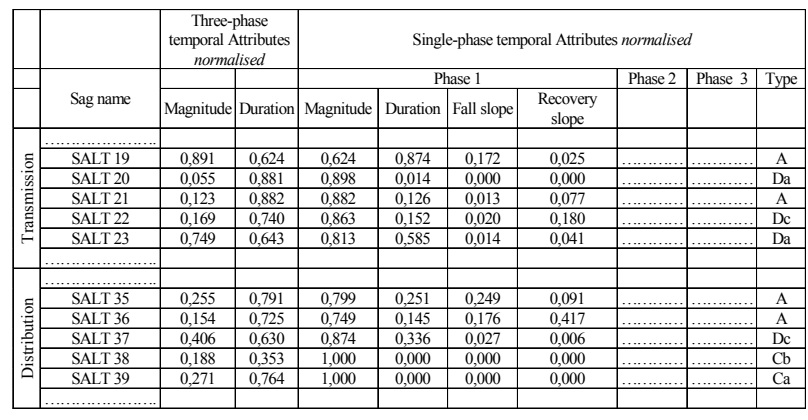

Table 2: Voltage sag case-base recorded in a $25 \mathrm{kV}$ substation

The example shows the comparison between a new sag (SALT 18) and the stored sags in order to retrieve the most similar one. The explained method is being applied. The voltage sag type is $\mathrm{Db}$, then the next step is to take from the case-base all the $\mathrm{Db}$ sag type and retrieve the most similar.

Table 3 show the results obtained after applying Manhattan distance method to temporal attributes. Table 4 show the results obtained after applying Dynamic Time Warping to characteristic voltage.

\begin{tabular}{|c|c|c|c|c|}
\hline Sag name & Type & Distance & Location & Origin \\
\hline SALT 18 & Db & 0 & Distribution & Damaged conductor \\
\hline SALT 1 & Db & 0.09392768 & Distribution & Damaged conductor \\
\hline SALT 2 & Db & 0.10518463 & Distribution & Damaged conductor \\
\hline SALT 3 & Db & 0.4104873 & Distribution & Damaged conductor \\
\hline SALT 4 & Db & 0.50878981 & Distribution & Damaged conductor \\
\hline SALT 5 & Db & 0.78833902 & Distribution & Damaged conductor \\
\hline SALT 6 & Db & 1.1639413 & Distribution & Damaged conductor \\
\hline SALT 7 & Db & 1.2964107 & Transmission & $\begin{array}{c}\text { Single phase trip. Successful } \\
\text { reclose in both end line. }\end{array}$ \\
\hline SALT 8 & Db & 1.5534047 & Transmission & $\begin{array}{c}\text { Single phase trip. Successful } \\
\text { reclose in both end line. }\end{array}$ \\
\hline SALT 9 & Db & 1.5934227 & Transmission & $\begin{array}{c}\text { Single phase trip. Successful } \\
\text { reclose in one end line. }\end{array}$ \\
\hline SALT 10 & Db & 1.6972648 & Transmission & $\begin{array}{c}\text { Single phase trip. Successful } \\
\text { reclose in both end line. }\end{array}$ \\
\hline SALT 11 & Db & 1.7295983 & Transmission & $\begin{array}{c}\text { Single phase trip. Successful } \\
\text { reclose in both end line. }\end{array}$ \\
\hline SALT 12 & Db & 1.779331 & Transmission & $\begin{array}{c}\text { Single phase trip. Successful } \\
\text { reclose in one end line. }\end{array}$ \\
\hline SALT 13 & Db & 2.0836669 & Distribution & Damaged conductor \\
\hline SALT 14 & Db & 2.1924684 & Distribution & Damaged conductor \\
\hline SALT 15 & Db & 2.4063196 & Distribution & Single phase trip \\
\hline SALT 16 & Db & 3.0208836 & Transmission & Damaged conductor \\
\hline SALT 17 & Db & 3.7465998 & Transmission & Single phase trip \\
\hline
\end{tabular}

Table 3: new sag and temporal attributes distances

For both, the first five sags retrieved are very similar to the new sag and also diagnostic is correct.

Figures 9 and 10 show three-phase voltage sags and their characteristic voltage. 
In figure 9a), 9b) and 9c), the new voltage sag and the most similar sags retrieved by both methods are presented.

In figure 9d) sag (SALT 13) seems to be very similar, however appears in the end of both retrieved tables. It is because in both methods magnitude is more relevant than voltage duration time. Whit Manhattan methods this kind of situations can be controlled with Wi. Figure 10a) and $10 \mathrm{~b}$ ), is a contrary case, these sags have different form but almost the same magnitude.

\begin{tabular}{|c|c|c|c|}
\hline Sag name & DTW Distance & Location & Origin \\
\hline SALT 18 & 0.0000000000 & Distribution & Damaged conductor \\
\hline SALT 4 & 0.0000018196 & Distribution & Damaged conductor \\
\hline SALT 1 & 0.0000022651 & Distribution & Damaged conductor \\
\hline SALT 5 & 0.0000039876 & Distribution & Damaged conductor \\
\hline SALT 6 & 0.0000065165 & Distribution & Damaged conductor \\
\hline SALT & 0.0000067012 & Distribution & Damaged conductor \\
\hline SALT 12 & 0.0000262950 & Transmission & Single phase trip. Successful reclose in one end line. \\
\hline SALT 7 & 0.0002201800 & Transmission & Single phase trip. Successful reclose in both end line. \\
\hline SALT 9 & 0.0002363000 & Transmission & Single phase trip. Successful reclose in one end line. \\
\hline SALT 17 & 0.0008392900 & Transmission & Single phase trip \\
\hline SALT 11 & 0.0008832800 & Transmission & Single phase trip. Successful reclose in both end line. \\
\hline SALT 8 & 0.0010595000 & Transmission & Single phase trip. Successful reclose in both end line. \\
\hline SALT 10 & 0.0013529000 & Transmission & Single phase trip. Successful reclose in both end line. \\
\hline SALT 6 & 0.0015008000 & Distribution & Damaged conductor \\
\hline SALT 13 & 0.0015247000 & Distribution & Damaged conductor \\
\hline SALT 16 & 0.0015721000 & Transmission & Damaged conductor \\
\hline SALT 14 & 0.0018798000 & Distribution & Damaged conductor \\
\hline SALT 15 & 0.0092643000 & Distribution & Single phase trip \\
\hline
\end{tabular}

Table 4: new sag and DTW application

Look in table 4, that the first five sags retrieved by DTW have two or three orders of magnitude compared to the other sags, it permit to say that DTW has a important discrimination capabilities.
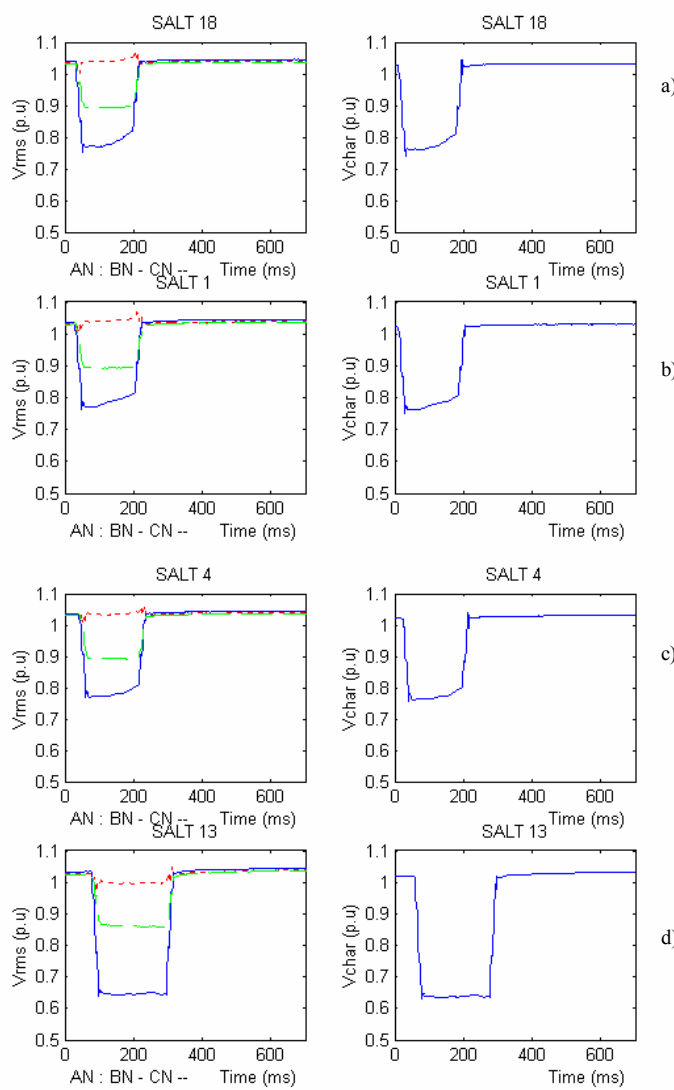

Figure 9 New sag and the most similar retrieved
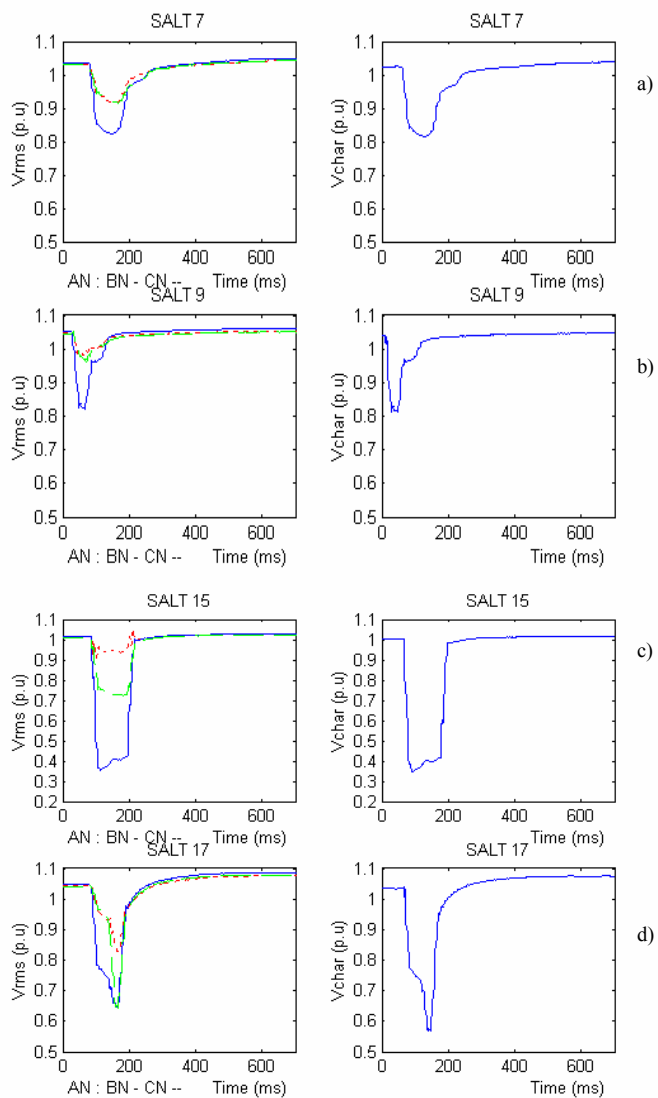

Figure 10: Sags comparison

Retrieved sags SALT 15 and SALT 17 are the lees similar because they have a considerable magnitude, short duration and different shape.

\section{Conclusions}

The increase of measures of voltage sags abstracted from the waveshape allows to compare sags in order to diagnostic the origin of the fault.

Two methods have been proposed to define similarities between sags, taking into account both quantitative and qualitative attributes.

\section{Future Work}

The proposed methods most be tested with more dates. In the first method, Wi can be configured with values that allow to improve the retrieve criteria.

PN-factor can also be considered as attribute to apply Dynamic Time Warping.

\section{Acknowledgements}

This work has been partially supported by Spanish government and FEDER funds (SECSE, DPI2001-2198) and a contract between UdG and ENDESA Distribution. 


\section{References}

[1] N. Kagan. "Influence of RMS variation measurement protocols on electrical system performance indices for voltage sags and swells" Proceedings. Ninth International Conference on, Vol. III, pp. 790 -795, 2000.

[2] L.D. Zhang, M.H.J. Bollen, A method for characterizing unbalanced voltage dips (sags) with symmetrical components, IEEE Power Engineering Letters, July 1998.

[3] Electromagnetic Compatibility (EMC), Part 4. Testing and measurement protocols. Section 11. Voltage dips, short interruptions and voltage variations immunity tests. IEC document 61000-4-11.

[4] Recommended practice for evaluating electric power system compatibility with electronic process equipment, IEEE Std. 1346-1998.

[5] Measurement guide for voltage characteristics, UNIPEDE report 23002 Ren 9531.

[6] M.H.J.Bollen, P. Wang, N. Jenkins, Analysis and consequences of the phase jump associated with a voltage sag, Power System Computation Conference, Dresden, Germany, August 1996.

[7] M.H.J. Bollen, The influence of motor reacceleration on voltage sags, IEEE Transactions on Industry Applications, vol.31, 1995, p.667-674.

[8] Schank R.C., "Dynamic Memory. A theory of reminding and learning in computers and people. Cambridge University Press, Cambridge 1982.

[9] Wilke W., Bergmann R., "Technques and Knowledge used for adaption during case-based problem solving", in LNAN 1416, vol.2, Springer, 1998, pp: 497-506.

[10] Aamodt A. And Plaza, E., "Case-based Reasoning : Foundational Issues, Methodological Variations, and system approaches", Artificial Intelligence Communications, IOS Press, Vol : 1, 1994, pp: 39-59.

[11] Langseth H., Aamodt. A., winnem O.M., "Learning Retrieval Knowledge from data", IJCAI'99 Workshop ML-5: Automating the construction of Case-Based Reasoners, Anand, Aamodt and Aha Eds., Stockholm 1999, pp:77-82.

[12] J. J. Mora, D. Llanos, J. Meléndez "Classification of Sags Measured in a Distribution Substation based on Qualitative and Temporal Attributes", Proceedings CIRED 2003.

[13] J. J. Grainger, W. D. Stevenson, Análisis de Sistemas de Potencia, McGraw-Hill, México (1998), pp. 441-497.

[14] IEEE Std 1159-1995, "IEEE recommended practice for monitoring electric power quality", IEEE Standards Coordinating Committee 22 on Power Quality, USA (1995), pp. 12

[15] Colomer J.; Meléndez J.; Gamero, F.I, "Pattern recognition based on episodes and DTW. Application to diagnosis of a level control system", 16th International Workshop on Qualitative Reasoning, QR 2002, pp. 37-43. 